\title{
PIC: Enable Large-scale Privacy-preserving Content-based Image Search on Cloud
}

\author{
Lan Zhang ${ }^{1}$, Taeho Jung ${ }^{2}$, Puchun Feng ${ }^{1}$, Kebin Liu ${ }^{1}$, Xiang-Yang Li $^{1}, 2$, Yunhao Liu ${ }^{1}$ \\ ${ }^{1}$ School of Software Engineering, Tsinghua University \\ , ${ }^{2}$ Department of Computer Science, Illinois Institute of Technology
}

\begin{abstract}
High-resolution cameras produce huge volume of high quality images everyday. It is extremely challenging to store, share and especially search those huge images, for which increasing number of cloud services are presented to support such functionalities. However, images tend to contain rich sensitive information (e.g., people, location and event), and people's privacy concerns hinder their readily participation into the services provided by untrusted third parties.

In this work, we introduce PIC: a Privacy-preserving large-scale Image search system on Cloud. Our system enables efficient yet secure content-based image search with fine-grained access control, and it also provides privacy-preserving image storage and sharing among users. Users can specify who can/cannot search on their images when using the system, and they can search on others' images if they satisfy the condition specified by the image owners. Majority of the computationally intensive jobs are outsourced to the cloud side, and users only need to submit the query and receive the result throughout the entire image search. Specially, to deal with massive images, we design our system suitable for distributed and parallel computation and introduce several optimizations to further expedite the search process.
\end{abstract}

We implement a prototype of PIC including both cloud side and client side. The cloud side is a cluster of computers with distributed file system (Hadoop HDFS) and MapReduce architecture (Hadoop MapReduce). The client side is built for both Windows OS laptops and Android phones. We evaluate the prototype system with large sets of real-life photos. Our security analysis and evaluation results show that PIC successfully protect the image privacy at a low cost of computation and communication.

\section{INTRODUCTION}

As on-board cameras get more and more popular, numerous high-resolution photos are generated everyday, which makes storing, sharing and especially searching large-scale images become challenging issues. There are an increasing number of service providers supporting cloudbased image services, e.g., Amazon Cloud Drive, Apple iCloud, Cloudinary, Flicker and Google. Content-based image search is a core functionality for a variety of image applications, such as personal image management, criminal investigation using crowd-sourced photos (e.g., the Boston Marathon investigation [1]) and medical image study and diagnosis [2], [3]. It draws many attentions from both industry (e.g., Google) and academics (e.g., [4], $[5])$. However, there are a lot of private information in images and the cloud could be untrustworthy [6], which hinder the wide adoption of those useful image services. While leveraging the power of cloud and crowdsourcing, image services should be provided in a privacy-preserving way. Besides, the image owner also requires the right to determine who is valid to search and access his images.

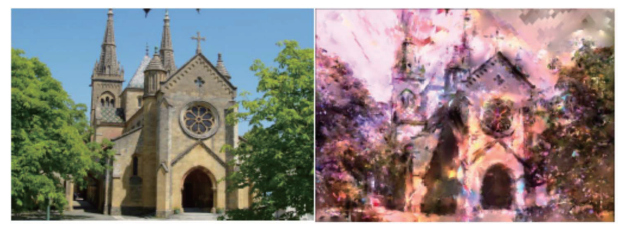

(a) Compare original image and reconstructed image using SIFT feature vector. [7]

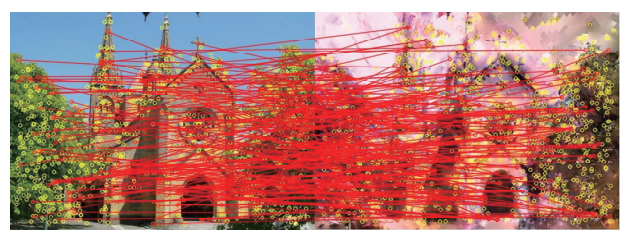

(b) Feature vector detection and matching results between original image and reconstructed image.

Fig. 1. Comparison between original image and images reconstructed from feature vectors.

The state-of-the-art image search systems typically use feature descriptors of images to measure their content similarity, e.g., SIFT [8]. Feature descriptors are sets of high-dimension feature vectors, e.g., 128-dimension for SIFT [8], extracted from interest points of the images (Fig. 1). Those feature descriptors are distinctive and show good accuracy for image search. One image is usually described by hundreds of feature vectors, then the millions of photos uploaded to the cloud imply that billions of feature vectors need to be processed. Therefore, it is necessary to introduce some optimization techniques such as indexing or distributed computing to accelerate the search process. Using feature vectors, many indexing mechanisms are designed to facilitate large-scale image search, e.g., [9], [5] and [10]. But most of them did not support image privacy protection or assumed that the feature vectors do not reveal useful information about the original images. However, recent research shows that an image can be approximately reconstructed based on the output of a blackbox feature descriptor software such as those classically used for image indexing [7], [11]. As presented in Fig. 1, the image reconstructed using SIFT 
feature vectors appears quite similar as the original image, and shows a good matching result with the original one. Even quantized binary feature vectors expose the content of an image to some extent [12]. Those methods are entirely automatic and much better reconstruction can be achieved with user interaction, which arouse great concerns on the image content privacy in the image indexing and search systems.

Recently, some facts show that people pay more and more attention to the privacy of their images, and the increasing worry about privacy may be the key point in the future cloud-based image services. For example, a smartphone app KeepSafe [13] hides images and videos from unauthorized users by simply encrypting image folders with a PIN code, and it owns 13 million users and gained an investment of 2 millions dollars. Furthermore, the face search functionality of Facebook has been abandoned for two years due to the privacy concern from users and governments [14].

As a result, massive image need efficient yet private content-based search functions urgently. Existing largescale image indexing and search systems usually ignore the privacy issue and do not support privacy protection mechanisms. Some systems tag images with keywords and metadata, and search on encrypted keywords, e.g., [15]-[18]. But keyword-based search methods cannot be adopted for content-based image search, because they only search the occurrences of encoded tags in an exact match manner and cannot measure the distance between highdimension feature vectors. Moreover, they usually reveal the search results to the cloud. To achieve content-based image search, we need to measure the distance between pairs of feature vectors as well as to protect the vectors and search results. This problem is especially challenging when we require no interaction with the image owner during the whole search process and the image data are extremely large (e.g., with millions of feature vectors). Traditional secure multi-party computation (SMC) [19], [20], verifiable computation [21] or simple homomorphic encryption [22], [23] support privacy-preserving vector distance computation. But they cannot be the solution to the large-scale image search problem. On one hand, the garbled circuit's size is exponentially greater than the size of the input, and the input size in an image search is often very large (e.g., thousands of 128-dimensional feature vectors of real values), so the communication and computation overhead is beyond practicality. On the other hand, a simple homomorphic method will allow the decrypter of the final result to also decrypt the individual ciphertexts. Moreover, it requires rounds of interactions between the querier, data owner and cloud server, which means the image owners need to always stay online to react to image queries, which is not practical either. Facing these challenges, we employ the multi-level homomorphic encryption (Xiao et al [24]) as a building block to design an efficient non-interactive image search system.

The main contributions of this paper can be summarized as follows:

1) We propose a novel system (PIC) and techniques to enable private content-based image search upon large- scale encrypted images with untrusted servers. Our system outsources the majority of the search job to the cloud side, but neither the query result nor the query itself is revealed to the cloud. What's more, during the search, no interaction is required between the data owner and the querier or the cloud.

2) We design our private search compatible with the standard image search to guarantee the search accuracy. We also make it suitable for distributed and parallel computation to enable efficient large-scale image search. Several optimizations are introduced to further expedite the search process.

3) We implemented a prototype including both cloud side and client side. The cloud is a cluster of computers with distributed file system (Hadoop HDFS) and MapReduce architecture (Hadoop MapReduce). We evaluate our system using large sets of highly diverse real-life photos. The security analysis shows that PIC successfully protect the image privacy, and the evaluation shows the efficiency of our system, which is capable to be used in a wide range of platforms including resource-bounded ones.

Roadmap. The rest of this paper is organized as follows. Section II reviews the image search model and Section III introduces the building blocks of PIC. Section IV gives an overview of our system framework. Section V presents the details of our basic design, then we discuss the bottleneck of system performance and refine system design in Section VI. We show the security of PIC in Section VII. To evaluate the practicality, we present comprehensive experiments in Section VIII. Section IX discusses the related work, and Section X concludes this paper.

\section{BACKGROUND ON LARGE-SCAle ImAGE SEARCH}

We address the problem of efficient large-scale image search with untrusted cloud servers. Different from existing work focusing on the search efficiency, we also consider the image privacy (the image itself and its feature descriptors) of the image owner and the query privacy (the query image content and the query result) of the querier. To guarantee the search accuracy, we employ the state-of-the-art largescale image search model in the computer vision field. In this section, we briefly review the image search model to enhance the understanding of our system.

\section{A. Image Feature Descriptor}

In the field of computer vision, many approaches have been proposed to search similar images. Feature descriptor is widely adopted for image similarity measurement. Given an image $I$, interest points are detected and for each interest point one feature vector $\mathbf{x}_{i}$ is extracted to describe the visual characteristics around this point. The feature descriptor of an image consists of all feature vectors extracted from it, denoted as $\mathbf{X}:=\left\{\mathbf{x}_{1}, \ldots, \mathbf{x}_{\alpha}\right\}$. To achieve robust and fast image description, different types of descriptors are propsed, e.g., SIFT [8] and SURF [25]. For a specific type of descriptor, feature vectors are usually of the same dimension, e.g., the feature vector of SIFT descriptor is 128-dimension. 


\section{B. Voting-based Search Model}

Many modern content-based image retrieval systems manage millions of images, i.e., billions of high-dimension feature vectors. Facing the big image database, the stateof-the-art solutions (e.g., [26], [9] and [27]) usually search similar images as follows: fist searching similar vectors for feature vectors of the querying image in an index structure, and then obtains matched images using a voting-based model.

Given a query descriptor $\mathbf{X}:=\left\{\mathbf{x}_{1}, \ldots, \mathbf{x}_{\alpha}\right\}$ (descriptor of the query image $I_{x}$ ), and a set of of descriptors $\left\{\mathbf{Y}^{1}, \cdots, \mathbf{Y}^{N}\right\}$ of images in the DB, where $\mathbf{Y}^{n}$ is the descriptor of the $n$-th image, the voting-based search model works as follows:

1) Let the score of each image in DB be $S^{n}$, and initialize all scores to 0 .

2) For each feature vector $\mathbf{x}_{i}$ of $\mathbf{X}$ and each feature vector $\mathbf{y}_{j}^{n}$ in DB, the score $S^{n}$ is increased by $S^{n}:=$ $S^{n}+\delta\left(\mathbf{x}_{i}, \mathbf{y}_{j}^{n}\right)$, where $\delta\left(\mathbf{x}_{i}, \mathbf{y}_{j}^{n}\right)$ is a matching function measuring the similarity between feature vectors $\mathbf{x}_{i}$ and $\mathbf{y}_{j}^{n}$ based on $k$-nearest neighbors ( $k$-NNs). Formally, the matching function is defined as

$$
\delta\left(\mathbf{x}_{i}, \mathbf{y}_{j}^{n}\right)= \begin{cases}1 & \text { if } \mathbf{y}_{j}^{n} \text { is a } k-\mathrm{NN} \text { of } \mathbf{x}_{i} \\ 0 & \text { otherwise }\end{cases}
$$

For the $k$-NNs search, the dissimilarity of feature vectors are typically measured by Euclidean distance.

3) By ranking the image scores, images with largest scores are selected as the matched images.

Facing hundreds of feature vectors of a query image and billions of feature vectors in DB, accurate $k$-NNs search is too expensive for most image search applications. Approximate search greatly improves the performance but suffers from a little loss of search accuracy. The most common way is indexing the massive feature vectors by partitioning them into groups via high-dimension clustering. Given a query feature vector, the system firstly finds the closest cluster representative and fetches the contents within this cluster into the memory; secondly, distances between the query vector and fetched vectors are computed to get the $k$-NNs. As a result, many clusters can be pruned quickly to accelerate the searching process.

\section{MapReduce Framework}

The large-scale high-dimension vector based image search can be very costly for both memory and CPU even with an index structure. MapReduce [28] is a software framework for the applications which process vast amounts of data (multi-terabyte datasets) on large clusters (thousands of nodes) of commodity hardware in a parallel, reliable and fault-tolerant manner. Exploiting the power of parallel computing of MapReduce, the image query can be handled within an acceptable response time [27].

\section{Multi-Level Homomorphic EnCRYPtion}

To empower the cloud to maintain the image index as well as search images in a privacy-preserving way, we employ an efficient multi-level homomorphic encryption
(HE) protocol presented by Xiao et al. [24]. The protocol is defined as follows:

Definition 1: The multi-level homomorphic encryption is defined by three algorithms (K,HE.E, HE.D), where $K$, HE.E and HE.D are the key generation, encryption and decryption algorithms, and it satisfies HE. $\mathrm{D}(\mathrm{HE} . \mathrm{E}(m, k))=$ $m$ given $k \leftarrow K\left(1^{\lambda}\right)$.

We carefully review the design and security proof of this protocol, and make sure it is correct. Considering it is not the contribution of this work, we omitted the detail explanation due to space limitation. Notably, the homomorphic encryption has the following good properties we want to utilize in our work:

Additive and Multiplicative Homomorphism: Their encryption has the following homomorphism:

$$
\begin{aligned}
\text { HE. } \mathrm{E}\left(m_{1}, k\right) \cdot \operatorname{HE} . \mathrm{E}\left(m_{2}, k\right) & =\mathrm{HE} . \mathrm{E}\left(m_{1} m_{2}, k\right) \\
\mathrm{HE} . \mathrm{E}\left(m_{1}, k\right)+\mathrm{HE} . \mathrm{E}\left(m_{2}, k\right) & =\mathrm{HE} \cdot \mathrm{E}\left(m_{1}+m_{2}, k\right)
\end{aligned}
$$

This also implies the homomorphism over any polynomial function $f$, i.e.,

$$
\begin{aligned}
& f\left(\mathrm{HE} \cdot \mathrm{E}\left(m_{1}, k\right), \mathrm{HE} \cdot \mathrm{E}\left(m_{2}, k\right), \cdots, \mathrm{HE} \cdot \mathrm{E}\left(m_{l}, k\right)\right) \\
& =\mathrm{HE} \cdot \mathrm{E}\left(f\left(m_{1}, m_{2}, \cdots, m_{l}\right), k\right)
\end{aligned}
$$

Key Conversion: If we have $k=\prod_{i} k_{i}$ for the key $k$, the encryption has the following property:

$$
\left(\prod_{i} k_{i}^{-1}\right) \cdot E(m, 1) \cdot\left(\prod_{i} k_{i}\right)=E\left(m, \prod_{i} k_{i}\right)=\mathrm{HE} . \mathrm{E}(m, k)
$$

This implies that one does not need to decrypt and reencrypt the message to alter the key of a ciphertext, which is very useful in our system design. Note that a randomizer is omitted for the sake of simplicity, so it is not possible to attack this encryption via brute-force search if the ciphertext size is large enough.

\section{A. Distance Calculation via $H E$}

Based on HE, we can conduct the distance calculation for two feature vectors $\mathbf{x}, \mathbf{y}$ on the ciphertexts as follows, where $\mathbf{x}(j)$ refers to the $j$-th dimension of the feature vector $\mathbf{x}$, and $\mathrm{D}(\mathbf{x}, \mathbf{y})=\mathrm{d}^{2}(\mathbf{x}, \mathbf{y})$.

$$
\mathrm{HE} . \mathrm{E}(\mathrm{D}(\mathbf{x}, \mathbf{y}), k)=\sum_{k}(\mathrm{HE} . \mathrm{E}(\mathbf{x}(j), k)-\mathrm{HE} \cdot \mathrm{E}(\mathbf{y}(j), k))^{2}
$$

Then, the distance calculation can be outsourced to anyone who does not know the key by giving him all the ciphertexts. Since the calculation is conducted on the ciphertexts, the outsourcing does not reveal information about feature vectors or the calculation output. Hereafter, we use the notation $\phi_{\mathrm{D}}(\cdot)$ to denote the function which conducts distance calculation given homomorphic ciphertexts of two vectors. That is:

$$
\phi_{\mathrm{D}}(\mathrm{HE} . \mathrm{E}(\mathbf{x}, k), \mathrm{HE} . \mathrm{E}(\mathbf{y}, k))=\mathrm{HE} . \mathrm{E}(\mathrm{D}(\mathbf{x}, \mathbf{y}), k) .
$$




\section{B. Fixed Point Representation}

The numeric type of feature vectors may be real number, but the homomorphic encryption used in this paper is based on large integers, therefore we need to use integers to represent real numbers first. In PIC, we use the fixed point representation ( [29]) to represent real numbers due to its simplicity when applying elementary arithmetic operations to it.

Given a real number $a$ and a fixed precision $p$, an $m+1$ dimension binary array $A$ satisfies the following in the fixed point representation with Two's Complement:

$$
\begin{cases}A[0]=0 \wedge \sum_{k=1}^{m} A[k] \cdot 2^{-k+p} \approx a & \\ A[0]=1 \wedge-\sum_{k=1}^{m-1}(A[k] \oplus 1) \cdot 2^{-k+p}-A[m] \cdot 2^{-k+m} \approx a & a<0\end{cases}
$$

The minimum unit of this representation is $2^{p-m}$ (i.e., precision), and the range of this representation is $\left(-2^{p-1}, 2^{p-1}\right)$. Then, we use the following integer to represent the real number $a$ (with some errors less than the minimum unit):

$$
\mathbf{f}(a)= \begin{cases}\sum_{k=1}^{m} A[k] \cdot 2^{m-k} & \text { if } A[0]=0 \\ -\sum_{k=1}^{m}(A[k] \oplus 1) 2^{m-k} & \text { if } A[0]=1\end{cases}
$$

which is the definition of signed integer with Two's complement.

table I. Fixed Point Representation Operations

\begin{tabular}{|c|c|}
\hline,+- & $\mathbf{f}(a \pm b)=\mathbf{f}(a) \pm \mathbf{f}(b)$ \\
\hline$\times$ & $\mathbf{f}(a \cdot b)=\mathbf{f}(a) \cdot \mathbf{f}(b) / 2^{m-1-p}$ \\
\hline
\end{tabular}

Note that the addition/subtraction $(x \pm y)$, multiplication $(x \cdot y)$ and the division $\left(\frac{x}{y}\right)$ are all elementary arithmetic operations closed in integer domain (i.e., $a / b$ is the quotient of $\frac{a}{b}$ ). We assume $m, p$ are pre-defined parameters based on the range and precision requirements of the application. For simplicity, we will omit the realinteger conversion and use normal arithmetic operations on real numbers in the following presentation, but the values must be converted to the fixed point representation and fixed point representation operation (Table I) should be applied in applications.

\section{System Overview}

We present the overview of our system design, threat model and the security assumption before further presenting design details.

\section{A. Architecture \& Entities}

Fig. 2 describes the flow of our system. The system is designed to let users store, share and search images via external cloud servers without conducting computationally heavy tasks. The entire outsourced computation is conducted on the ciphertexts directly, therefore users' image content privacy is preserved against cloud servers or other adversaries. Specifically, for the image owner our system protects his images, images' feature descriptors and search results (similarities) from unauthorized parties (including KA and CS); for the image querier, our system also protects the querying content from unauthorized parties. More precisely, we have the following entities in our system:

- Users: a user can be an image owner and an image querier simultaneously. An owner stores and shares his images with others by outsourcing them to the cloud servers, and a querier searches an image on the DB located at the cloud server side.

- Cloud Server (CS): The cloud server is in charge of majority of the computation and the storage throughout the system. Whenever a transaction request arrives, he processes the request by computing on the ciphertexts. CS could be any commercial cloud service providers who are willing to improve their services to attract more privacy-sensitive users.

- Key Agent (KA): The key agent manages various secret keys such that no one within the system (including himself) learn the final key used in the encryption. Majority of the transaction between users and CS is relayed by KA. KA could be any agent who is unlikely to collude with the CS or a specific user. For example, for crowd-sourced criminal investigation, KA can be a government-controlled agent; for medical image analysis, KA can be an authoritative medical organization; for personal image management, KA can be a reputable information security service provider.

\section{B. Threat Model}

The trusted party (TP) is introduced only to generate the keys, who could be an auditor or a notary public and is assumed to be fully trusted. However, the CS and the KA who conduct most of the computation may be motivated to infer useful information from the outsourced computation, and they are assumed to be semi-honest, i.e., they will follow the protocol specification in general, but will also try their best to harvest the content of the encrypted communication. In general, TP,CS and KA are well protected, so we do not consider compromise attack in this work. Also, although CS and KA are assumed to be semi-honest, the probability that both of them collude with a specific user is extremely small. Therefore, we assume that it is not possible to have a user who colludes with both CS and KA.

\section{Security Assumption}

Because we employ the homomorphic encryption in Xiao et al. [24] whose security rely on the assumption that prime factorization is hard, we also assume the following prime factorization is hard:

Definition 2: (Prime Factorization) A prime factorization problem is to solve all $p_{i}$ 's given a product of prime numbers $n=\prod_{i} p_{i}$.

\section{Basic System Design}

With the background and the system model, we are ready to present the detailed design of our system PIC. Our system supports the following four operations to serve the users: initialization, key generation \& policy 


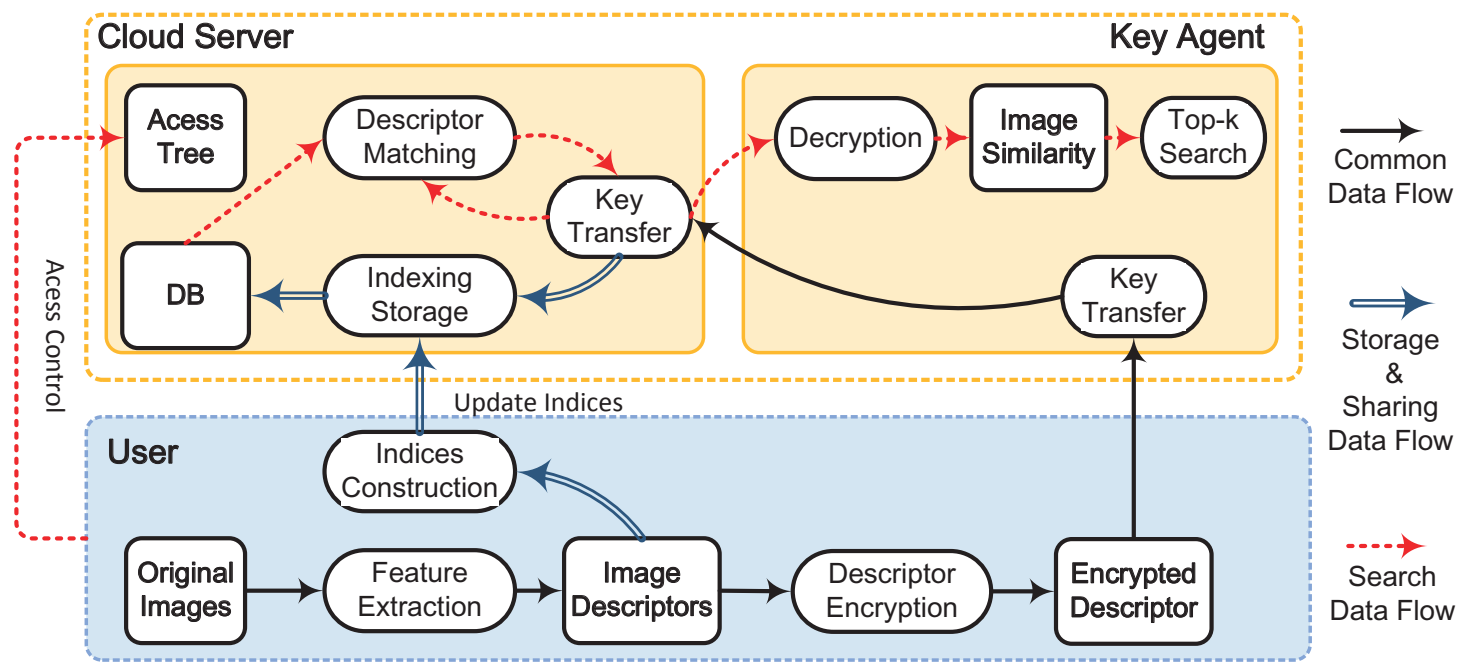

Fig. 2. PIC Architecture

announcement, image upload and privacy preserving image search with access control.

\section{A. Initialization}

Firstly, a TP picks the system parameter for the homomorphic encryption ( [24]) and publishes it. Then, TP generates a master key $k$ to be used in the homomorphic encryption, and he finds two random keys $k_{C S}, k_{K A}$ such that $k_{C S} k_{K A}=k$ and sends $k_{C S}$ and $k_{K A}$ to CS and $\mathrm{KA}$ respectively via secure channel.

\section{B. Key Generation $\&$ Policy Announcement}

Whenever a new user $u$ joins the system, TP generates three random keys $k_{u}, k_{u}^{\prime}, k_{u}^{\prime \prime}$ such that $k=k_{u} k_{u}^{\prime} k_{u}^{\prime \prime}$. Then, he gives $k_{u}^{\prime}$ to KA, $k_{u}^{\prime \prime}$ to CS and $k_{u}$ to the user $u$ via secure channel.

Then, the user defines the access policy which controls who can/cannot search on his images. The policy is described by an access tree as in CP-ABE [30], and it is uploaded to CS for further access control.

Submitting raw attributes to CS will reveal the user's identity information ( [31]). Therefore, the attributes as well as access policy should be masked before uploading. Note that the access control policy is used as a blackbox building block in our system, and here we present a simple policy which works with CP-ABE as a baseline method. When joining the system, every user describes his access policy with an access tree, but the attributes at the leaf nodes are replaced with the hashed values of the attributes. Whenever a querier wishes to search on a group of specified users or the entire DB, the querier submits his hashed attributes to CS. CS then matches these hashed attributes with the access policies in the DB to find out the group of users that the querier is valid to search on. We evaluate the practicality of the access policy by investigating a real social networking system (in SectionVIII-D), and our analysis shows that for most cases the simple access policy is sufficient and practical. For some special cases, there can be other better options for the access control (e.g., anonymous IBE with predicate encryption [32]) which achieves better anonymity, but this is not our main contribution, and we leave it as one of our future work to study.

\section{Image Upload}

Whenever a new user $u$ uploads some images, he first extracts the feature descriptors from them, and encrypts the descriptors using his key $k_{u}$ as follows:

$$
\mathrm{HE} . \mathrm{E}\left(\left\{\mathbf{X}_{i, 1}, \mathbf{X}_{i, 2}, \cdots\right\}, k_{u}\right)
$$

Then, the user needs to either create or update the index cluster for his feature descriptors by two phases. (1) Indices construction: cluster representatives are selected from feature vectors as centroids of clusters. (2) Clustering: other vectors are assigned to clusters. Various clustering techniques can be applied, and different clustering techniques have different performance and accuracy. One can simply use k-mean clustering to create the index of vectors. At each time the user wants to update the index, he will either re-construct the it or just incrementally append new representatives or nodes into the current clusters.

After the clusters are prepared, he appends references to the nodes in the cluster which points to the corresponding ciphertexts of feature vectors. Then, the raw index clusters are sent to CS. To reduce the communication overhead, the user can send only the change of the index cluster instead. Once he completes the update (or creation), the ciphertexts are sent to KA.

$\mathrm{KA}$, upon receiving the ciphertexts, conducts the following operation for every ciphertext to get the ciphertexts with altered key $k_{u} k_{u}^{\prime}$ :

$$
k_{u}^{\prime-1} \mathrm{HE} . \mathrm{E}\left(\left\{\mathbf{X}_{i, 1}, \cdots\right\}, k_{u}\right) k_{u}^{\prime}=\operatorname{HE} . \mathrm{E}\left(\left\{\mathbf{X}_{i, 1}, \cdots\right\}, k_{u} k_{u}^{\prime}\right)
$$

Then, KA sends the altered ciphertexts to CS.

CS conducts the following operation to get the final ciphertexts:

$$
\begin{aligned}
k_{u}^{\prime \prime-1} \mathrm{HE} . \mathrm{E}\left(\left\{\mathbf{X}_{i, 1}, \cdots\right\}, k_{u} k_{u}^{\prime}\right) k_{u}^{\prime \prime} & =\operatorname{HE} . \mathrm{E}\left(\left\{\mathbf{X}_{i, 1}, \cdots\right\}, k_{u} k_{u}^{\prime} k_{u}^{\prime \prime}\right) \\
& =\operatorname{HE} . \mathrm{E}\left(\left\{\mathbf{X}_{i, 1}, \cdots\right\}, k\right)
\end{aligned}
$$


Then, CS merges the user $u$ 's index cluster with the global one for his DB, but leaving a label to mark the owner of the cluster.

\section{Privacy-preserving Search with Access Control}

One image search has two phases: level-1 search and level-2 search. In the level-1 search, KA first finds out the cluster representative in the index cluster which is closest to the querying feature vector. Then, in the level-2 search, he finds out the $k$-nearest neighbors $(k-\mathrm{NNs})$ of the querying feature vector within the cluster.

\section{Level-1 Search}

When a querier $q$ wants to search an image, he first extracts the feature descriptor (i.e., a set of feature vectors) from the querying image. Then, he encrypts the feature descriptor $\mathbf{X}_{q}$ of the querying image with his key $k_{q}$ as HE.E $\left(\mathbf{X}_{q}, k_{q}\right)$, and submits the ciphertexts to KA. Then, $\mathrm{KA}$ alters the ciphertext to $\operatorname{HE} . \mathrm{E}\left(\mathbf{X}_{q}, k_{q} k_{q}^{\prime}\right)$ and sends them to CS. CS finally alters the ciphertexts to

$$
\text { HE.E } \begin{aligned}
\left(\mathbf{X}_{q}, k_{q} k_{q}^{\prime} k_{q}^{\prime \prime} k_{C S}^{-1}\right) & =\operatorname{HE} . \mathrm{E}\left(\mathbf{X}_{q}, k k_{C S}^{-1}\right) \\
& =\operatorname{HE} . \mathrm{E}\left(\mathbf{X}_{q}, k_{K A}\right)
\end{aligned}
$$

Besides, the querier also uploads his hashed attributes to CS. CS then searches all users' access policies and finds out the users that the querier is valid to search on their images. Then, CS computes the following altered ciphertexts, where $\left\{\mathbf{y}_{o}\right\}$ refers to the set of representative feature vectors (cluster leaders) in previously found owners' index clusters, and all $\left\{\mathbf{y}_{o}\right\}$ are stored in the form of ciphertexts encrypted by $k$ :

$$
\begin{aligned}
k_{C S} \operatorname{HE} . \mathrm{E}\left(\left\{\mathbf{y}_{o}\right\}, k\right) k_{C S}^{-1} & =\mathrm{HE} . \mathrm{E}\left(\left\{\mathbf{y}_{o}\right\}, k k_{C S}^{-1}\right) \\
& =\operatorname{HE} . \mathrm{E}\left(\left\{\mathbf{y}_{o}\right\}, k_{K A}\right)
\end{aligned}
$$

After all the altered ciphertexts are ready, CS computes the $\phi_{\mathrm{D}}(\cdot)$ function (Section III-A) for every pair of (HE.E $\left.\left(\mathbf{x}, k_{K A}\right), \operatorname{HE} . \mathrm{E}\left(\mathbf{y}, k_{K A}\right)\right)$, where $\mathbf{x} \in \mathbf{X}_{q}$ is a feature vector belonging to the descriptor of the querying image, and $\mathbf{y} \in\left\{\mathbf{y}_{o}\right\}$. Then, CS achieves the pairwise encrypted distances, which are sent to KA.

$\mathrm{KA}$ is able to decrypt the distances since the ciphertexts are encrypted under his key $k_{K A}$. After decrypting the distances, he finds out the nearest neighbor for every $\mathbf{x} \in \mathbf{X}_{q}$.

\section{Level-2 Search}

After finding the nearest neighbor among the representatives for every $\mathbf{x} \in \mathbf{X}_{q}$, KA further requests the distances between the $\mathbf{x}$ and all the vectors within the nearest neighbor's cluster.

Upon receiving the request, CS generates the following altered ciphertexts using the key conversion (Section III) and $\phi_{\mathrm{D}}(\cdot)$ function as aforementioned, where $\left\{\mathbf{y}_{c}\right\}$ is the set of vectors within the nearest neighbor's cluster:

$$
\left\{\left\{\operatorname{HE} . \mathrm{E}\left(\mathrm{D}(\mathbf{x}, \mathbf{y}), k_{K A}\right)\right\}_{\forall \mathbf{y} \in\left\{\mathbf{y}_{c}\right\}}\right\}_{\forall \mathbf{x} \in \mathbf{X}_{q}}
$$

Then, he sends these ciphertexts of distances as well as the image IDs associated with the feature vectors in the ciphertexs to KA. KA decrypts the ciphertexts and determines the $k$-NNs among $\left\{\operatorname{HE} . \mathrm{E}\left(\mathrm{D}(\mathbf{x}, \mathbf{y}), k_{K A}\right)\right\}_{\forall \mathbf{y} \in\left\{\mathbf{y}_{c}\right\}}$ for each $\mathbf{x} \in \mathbf{X}_{q}$. Based on the distances and the corresponding image IDs, he calculates the score $S^{n}$ of all images (Section II) appearing in the image IDs sent from CS and returns the image ID with the highest score to the querier. The querier then retrieves the encrypted image from the DB. One can further apply oblivious transfer (will be described in Section IX) to prevent the CS from inferring the query result by monitoring its memory access.

In this level-2 search, if CS does not find $k$ ciphertexts within one cluster, he also chooses the next nearest neighbor among the representatives and sends the corresponding ciphertexts to KA as well. This is repeated until he finds out at least $k$ ciphertexts to return to KA.

\section{System Refinements}

The basic system we proposed in the previous section works fine in some cases, e.g. people recognition in a face image collection, but its performance is degraded in more general cases as we will discuss below. Therefore, we present several improvements for our scheme to boost the performance, and finally formally describe the advanced scheme with these optimizations in this section.

\section{A. Dealing with Complicated Images}

A feature descriptor usually contains a set of highdimension feature vectors, e.g. 128 dimensions for SIFT. Since each dimension is a 64-bit real value, when we consider the encryption, each feature vector's size becomes $64 \mathrm{~KB}$ because each dimension is encrypted with a $4 \times 4$ matrix with 256 -bit integers. Then, the number of feature vectors in an image determines the size of its feature descriptor. For the face recognition, 9 feature vectors are enough to conduct an accurate search because face models are well developed. However, for complicated image with hundreds of feature vectors, the size of ciphertexts is not acceptable for many mobile devices. Therefore, we further optimize our system using more compact image descriptor to reduce the communication overhead.

We adopt frequency vector of visual words as image descriptor( [5], [33]). It quantizes the space of feature vectors to obtain a visual dictionary of size $n$. An image can be represented with the frequency histogram of visual words, by choosing the nearest word for each of its feature vectors. Then the feature descriptor is significantly reduced to one $n$-dimension frequency vector. The similarity between images can be computed by the scalar product of two frequency vectors.

To minimize the accuracy loss caused by quantizing the feature vector space to a discrete one, we weight the frequency vector with term frequency inverse document frequency (tf-idf, [33]). Based on tf-idf, given a dictionary of $n$ words and an image dataset of $N$ images, the weighted frequency vector of an image $I$ is $\mathbf{f}_{I}=\left(w_{1}, w_{2}, \cdots, w_{m}\right)$, and each weight $w_{i}$ is: $w_{i}=\frac{f_{i, I}}{n_{I}} \log \frac{N}{f_{i}}$, where $f_{i, I}$ is the 
frequency of the lexicographic (in the visual dictionary) $i$ th feature vector in the image $I, n_{I}$ is the total number of feature vectors in $I, f_{i}$ is the frequency of the $i$-th feature vector among $N$ images. The experimental results in peer work ( [33]) and our evaluation result (Section VIII-F) show that using this weighted visual word instead of exact feature vectors brings a negligible accuracy loss. Therefore, we use this weighted frequency vector with visual dictionary as our feature descriptor in our advanced scheme.

\section{B. Leveraging the Parallel Computation}

We further expedite the search process by applying the MapReduce framework. To do so, the search must support parallelism. Looking at the level-1 search and the level2 search, one can easily find both searches (finding the $\mathrm{NN}$ among the representatives and the k-NNs within the cluster) can be done by arbitrary number of mappers and one reducer. The ciphertexts of the feature vectors in DB and the querying vectors will be given to the mappers at CS, who conducts the homomorphic operations and the key modification to generate the ciphertexts of the distances. Then, they are sent to the reducer at KA who decrypts and sorts the distances.

However, there is a problem if our approach is directly implemented with a MapReduce framework. Unless the data is stored with a special format (e.g., bucketized), sorting limits the number of reducers to one because the mappers do not emit the (key,value $)=($ image ID, distance $)$ pairs in a sorted order. However, since the sorting order depends on the querying feature vector, the number of reducers will be always 1 . This is a great bottleneck since the reducer needs to wait for all (key,value) pairs emitted from all mappers before it continues to sort. To solve this bottleneck, we can return all the neighbors within a certain threshold distance instead of the exact NN in the level-1 search or the k-NNs in the level-2 search. By doing so, we can theoretically have arbitrary number of reducers to finish the search task in a parallel manner. If the threshold is chosen such that not enough results are found, one can easily use a binary search manner to adjust the threshold until enough results are found for further processing.

\section{Choosing a Good Clustering Method}

In fact, the clustering in the image upload operation heavily affects the performance and the accuracy, especially when it works with the MapReduce framework. Extended cluster pruning approach with recursion [10], [34] provides a promising way for efficient clusters construction and queries processing, and can be fitted appropriately in the MapReduce framework. Here we briefly review this approach and apply it in the advanced scheme.

Given a set $\mathbf{V}=\left\{\mathbf{v}_{1}, \mathbf{v}_{2}, \ldots, \mathbf{v}_{N}\right\}$ of $N$ vectors in $d$-dimension space and a positive integer $L$. The cluster pruning based approach searches $k$-nearest neighbors for a query vector $\mathbf{q}$ via two phases: preprocessing and query processing. During the preprocessing, $C$ vectors are chosen as representatives at random and denoted by $\mathbf{r}_{1}, \ldots, \mathbf{r}_{C}$. $C$ is determined by $C=N / S$, where $S$ is the target size of each cluster. The $C$ representatives are organized in a multi-level hierarchy of $L$ levels. Each other vector in $\mathbf{V}$ traverses the tree and is attached to its closest representatives at the bottom of the tree. The vectors are partitioned into $C$ clusters $\mathbf{C}_{1}, \ldots, \mathbf{C}_{C}$ with a logarithmic complexity. The clusters will be stored on a hard disk while the tree of representatives is small enough to be fitted in a memory, which can be processed efficiently by a mapper. Hereafter, we denote the representative of each cluster as $\mathbf{l}_{i}$, which is also called as the leader of this cluster.

During the query processing, by navigating down the tree of cluster leaders, the nearest leader of a query vector $\mathbf{q}$ is searched. Then the corresponding bottom cluster is fetched and the $k$-NNs for $\mathbf{q}$ are sought inside this cluster. Obviously, the $k$-NNs for $\mathbf{q}$ within the cluster is not always the global $k$-NNs of $\mathbf{q}$ among all feature vectors. Then, to improve the search accuracy, one may leverage the state-ofthe-art soft-assignment and multi-probe techniques. The soft-assignment allows each vector to be assigned to its $\alpha$ nearest representatives. The multi-probe allows each search to probe $\beta$ closest clusters simultaneously to search the overall $k$-NNs among these clusters.

\section{Advanced Scheme}

Only the image upload and the privacy-preserving image search with access control will be changed in the advanced scheme. The changed operations are as follows:

1) Image Upload: The user $u$ firstly extracts the feature vectors from every image that he wants to upload to CS. Then, he uses $k$-mean clustering to find the $k$ clusters among all his vectors, and sets all the centroids as elements in the visual dictionary of size $k$. Hereafter, we use $\mathbf{D}_{u}$ to denote user $u$ 's dictionary. After $\mathbf{D}_{u}$ is generated, user $u$ also calculates the $k$-dimension weighted frequency vector of each image $I$ as $\mathbf{f}_{I}$, and uses CP-ABE to encrypt all parameters in the weight function $\left(\left\{f_{i, I}, f_{i}\right\}_{i}, N\right)$ as well as $\mathbf{D}_{u}$ :

$$
\operatorname{ABE} . \mathrm{E}\left(\mathbf{D}_{u},\left\{f_{i, I}, f_{i}\right\}_{i}=1, \cdots, k, N\right) \text {. }
$$

User $u$ sends this ciphertext to CS, and encrypts all weighted frequency vectors as: $\operatorname{HE} . \mathrm{E}\left(\left\{\mathbf{f}_{I}\right\}_{I}, k_{i}\right)$. Then, he uses aforementioned extended cluster pruning approach with recursion to construct an index tree of all $\mathbf{f}_{I}$ 's, where each node contains the reference to the corresponding encrypted vector. The raw index tree is sent to CS (if only updated, only the changed parts are sent to CS), and the encrypted ciphertexts are sent to KA.

KA, upon receiving the ciphertexts, conducts the key modification to alter the ciphertexts to:

$$
\operatorname{HE} . \mathrm{E}\left(\left\{\mathbf{f}_{I}\right\}_{I}, k_{u} k_{u}^{\prime}\right) .
$$

Then, KA sends the new ciphertexts to CS, who conducts another key modification to achieve the final ciphertexts:

$$
\operatorname{HE} . \mathrm{E}\left(\left\{\mathbf{f}_{I}\right\}_{I}, k_{u} k_{u}^{\prime} k_{u}^{\prime \prime}\right)=\operatorname{HE} . \mathrm{E}\left(\left\{\mathbf{f}_{I}\right\}_{I}, k\right) .
$$

2) Privacy-preserving Search with Access Control: Same as the basic scheme, we also have two phases in a search. 


\section{Level-1 Search}

When a querier $q$ wants to search an image, he first extracts the feature vectors from the querying image. Then, he retrieves the ABE.E $\left(\mathbf{D}_{o},\left\{f_{i, I}, f_{i}\right\}_{i}=1, \cdots, k, N\right)$ 's of all image owner $o$ 's that he wants to search on, and decrypts the dictionaries as well as the parameters with his attributes. Then, he looks up each dictionary to create a frequency vector of his querying image for each $\mathbf{D}_{o}$. Then, he calculates $m$ weighted frequency vectors with the decrypted parameters, which are encrypted as: HE.E $\left(\{\mathbf{f}\}_{\text {all owners }}, k_{q}\right)$. This is relayed by KA (after altering the key to $k_{q} k_{q}^{\prime}$ ) and finally arrives CS who finally alters the key to $k_{q} k_{q}^{\prime} k_{q}^{\prime \prime}=k$. Then, CS finds out the index trees of the users that querier $q$ wishes to search on, and conducts the following key modification, where $\left\{\mathbf{y}_{o}\right\}$ refers to the set of frequency vectors referenced (via ciphertext) by the cluster leaders in those index trees:

$$
\text { HE.E }\left(\left\{\mathbf{y}_{o}\right\}, k k_{C S}^{-1}\right)=\operatorname{HE} . \mathrm{E}\left(\left\{\mathbf{y}_{o}\right\}, k_{K A}\right) \text {. }
$$

Then, CS computes the $\phi_{D}(\cdot)$ function for every pair of (HE.E $\left(\mathbf{x}, k_{K A}\right)$, HE.E $\left.\left(\mathbf{y}, k_{K A}\right)\right)$ where $\mathbf{x}$ is the frequency vector of the querying image, and $\mathbf{y} \in\left\{\mathbf{y}_{o}\right\}$. The outputs of the function are the pairwise encrypted distances, which are sent to KA.

KA proceeds with MapReduce framework. He sends the ciphertexts to his mappers, each mapper decrypts the distances and emits the (key,value $)=($ image ID,distance $)$ to his reducers, and the reducers find out the distances above a threshold $\theta$, which corresponds to the distances of x's nearest neighbors.

\section{Level-2 Search}

After finding the NNs, KA further requests the distances between $\mathbf{x}$ and all vectors under those $\mathrm{NNs}$ in the index trees.

Upon receiving the request, CS generates the encrypted distances using the key modification as well as the $\phi_{D}(\cdot)$ function. They are transmitted to KA who sends those ciphertexts to his mappers to let them decrypt the distances. The (image ID,distance) pairs are sent to his reducers, and they finally find out the images whose distances are above another threshold $\theta^{\prime}$.

\section{SECURITy AnALYsis}

The security of a system is given by the security of its weakest link. Since the participating adversaries are more powerful than non-participating adversaries and colluding adversaries are more powerful than a single adversary, we analyze the security of our system in the worst case scenario: colluding participating adversaries, including CS, $\mathrm{KA}$ and users (TP is fully trusted). Note that CS and KA do not collude with each other, and a user can collude with at most one party of CS and KA. Therefore, we have the following two cases: colluding user and CS; colluding user and KA.

It is already formally proved in [24] that the homomorphic encryption is secure against colluding user \& CS or colluding user \& KA, hence no party in the system can infer the plaintext from the ciphertext without knowing the key. We further analyze the extra information leakage in our system.

\section{Colluding user and KA}

Colluding user and KA only learn $k_{u}, k_{u}^{\prime}$ during the key generation, index construction or the index update. During the image search, KA receives a number of encrypted distances HE.E $\left(\mathrm{D}(\mathbf{x}, \mathbf{y}), k_{K A}\right)$ which are encrypted with $k_{K A}$. Then, KA can decrypt the distances, and the colluding user (a querier in this case) will know all the distances between the encrypted feature vectors in the database and his querying feature vectors. However, no ciphertexts of vectors are sent to KA or user. Therefore, the user only learns a number of distances, which is not useful to infer the images in CS's database. All the user gets is only the query result, which is the ciphertext of the matched image. Therefore, colluding user and CS do not gain useful information except the valid search result.

\section{Colluding user and CS.}

Colluding user and CS only learn $k_{u}, k_{u}^{\prime \prime}$ during the key generation. During the index forest construction and the index update, CS may share the ciphertext

HE.E $\left(\left\{\mathbf{X}_{i, 1}, \mathbf{X}_{i, 2}, \cdots\right\}, k_{u} k_{u}^{\prime}\right)$ received from KA with user, from which the user can use the key modification to achieve the ciphertext HE.E $\left(\left\{\mathbf{X}_{i, 1}, \mathbf{X}_{i, 2}, \cdots\right\}, k_{u}^{\prime}\right)$. However, since $k_{u}^{\prime}$ is unknown to both parties, it is not possible to infer $\mathbf{X}_{i, 1}, \mathbf{X}_{i, 2}, \cdots$ from the ciphertexts. During the image search, CS may also share the ciphertexts of the distance with the user, but neither CS nor the user is able to infer the distance from the ciphertext since none of them know the key $k_{K A}$.

\section{IMPLEMENTATION AND EVALUATION}

In this section, we first present the implementation of PIC and the datasets used in the experiments, then we evaluate the performance of our basic scheme using SIFT feature vectors and advanced scheme using weighted frequency vector comprehensively. For simplicity, in the following statement, we denote the basic scheme as PICsfv and the advanced scheme as PIC-wfv.

\section{A. System Implementation}

Our implementation of PIC includes both both cloud side and client side. On the cloud side, each of KA and CS consists of a cluster of computers with distributed file system (Hadoop HDFS) and MapReduce architecture (Hadoop MapReduce). In the experiments, we use four PCs with Intel Core i3-3240 CPU (3.4GHz) and 4G RAM for each cluster. Each cluster has one name node and three data nodes, which is a small but full-featured data center to demonstrate our design, and the performance can be greatly improved when using a large data center [35], [36]. On the client side, we implement our system for both Windows OS laptops and Android phones. In the experiments, we use a laptop (ThinkPad X1) with Intel Core i7-2620M CPU $(2.7 \mathrm{GHz})$ and $4 \mathrm{~GB}$ RAM, and a mobile phone (HTC G17) with $1228 \mathrm{~Hz}$ CPU, 1GB RAM. There is also a trusted party (TP) in charge of key generation. TP is a single PC with the same hardware as the node of the cluster. 


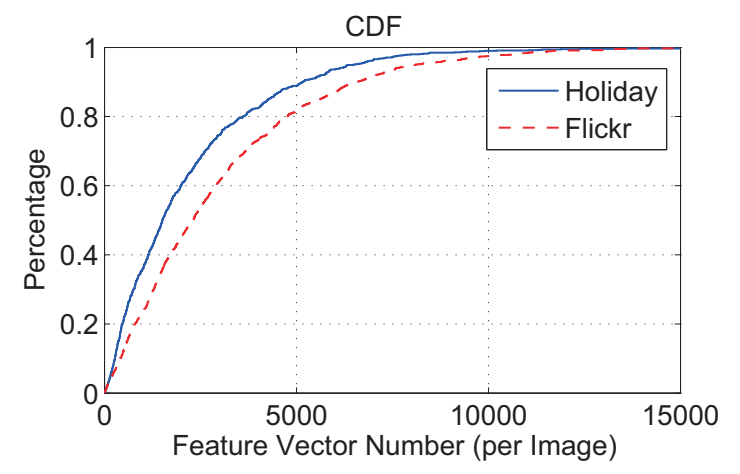

Fig. 3. CDF of feature vector number of images from two datasets.

We implement attribute based access control with the PBC (Pairing-Based Cryptography) library [37]. We develop all other cryptographic components are implemented by Java, including the fix point arithmetic and the multi-level homomorphic encryption [24]. We use three commonly used descriptors to evaluate the practicality of our system, including 128-dimension SIFT descriptor [8], 64-SURF and 128-SURF [25]. Nevertheless, our schemes are also compatible with other image descriptors. The descriptor extraction is implemented using the OpenCV library for Window and Android. Due to the space limitation, in this paper, we only present the results of the most popular descriptor, 128-SIFT, and 64-SURF and 128SURF achieve similar performance as that using 128-SIFT.

\section{B. Image Collections and Queries}

To explore the performance of our approach in reallife image applications, we evaluate our system with two popular image datasets.

- INRIA Holiday dataset (Holiday) [26] contains 1491 personal holidays photos in high resolution (most are $2560 * 1920)$. There are 6767563 SIFT feature vectors of dimensionality 128 extracted from those images. The dataset contains 500 image groups, each of which represents a distinct scene or object. For the search experiments, the query is a photo of a scene, and the goal is to return other $k$ photos of this scene.

- Flickr image dataset (Flickr1M) [38] contains one million diverse images from Flickr with 1.4 billion precomputed SIFT feature vectors in total. For the search experiments, the query is randomly selected images, and $k$ most similar images are returned.

We analyze the feature vector number of each image in two datasets. As shown in Fig. 3, The mean feature vector number is 2,200 and 2,988 for Holiday and Flickr1M respectively. Half images have less than 2000 feature vectors and $80 \%$ images have less than 5000 feature vectors.

We evaluate PIC-sfv using SIFT feature vectors from both datasets. For the evaluation of PIC-wfv, 1000 visual words are learned from $6 \mathrm{~K}$ randomly selected images of Flickr1M as the vocabulary. And a 1000-dimension weighted frequency vector is generated for each image by clients.

\section{Parameter Selection}

Before we present our evaluation results, we first discuss the selection of our experiment parameters.

1) Search Parameters Selection: There are three parameters governing the computation, communication and search performance for both schemes, including: (1) The number $C$ of created clusters, which determines the delay, overhead and accuracy of a search. Larger $C$ results in a longer clustering procedure and lower search accuracy, but a smaller search delay. There are some analysis on the parameter selection for optimal search quality [10], [34], and we follow them to set $C=\sqrt{N}$, resulting in $2 \sqrt{N}$ homomorphic distance calculations, where $N$ equals number of feature vectors for PIC-sfv and number of weighted frequency vectors for PIC-wfv. (2) The number $k$ of nearest neighbors, which influences the search accuracy. Voting-based methods are not very sensitive to $k$ for large collections [5], and we set $k=5$ in our evaluation, which produces good search results (Section VIII-F). (3) The work nodes number $N_{\text {node }}$ in the parallel computation. Existing work such as [35] and [36] have studied the performance gain as the number of work nodes increases. Based on those work, it is easy to estimate the performance of our system with more computing nodes.

For PIC-wfv, another key parameter is the size of the visual vocabulary $v$. Larger $v$ yields more accurate search result. It also determines the dimension of the weighted frequency vector for each image. As a result, the computation and communication cost increase linearly with $v$. Based on the ground truth of Holiday, we set $v=1000$ to optimize the search accuracy (93.4\%) with acceptable overhead.

2) Security Parameters Selection: For the multi-level homomorphic encryption, there are two parameters $\lambda$ and $m$ governing the security level and overhead of the system. The system can withstand an attack with up to $m \ln \operatorname{ploy}(\lambda)$ chosen plaintexts, while the communication cost increases linearly with $m \lambda$ and computation cost increases exponentially with $m \lambda$. As a result, there is a tradeoff between security and efficiency. In our experiments, we choose $m=2$ and $\lambda=128$, which allows $m \ln \lambda^{10} \sim 97$ plaintext attacks with good computation and communication cost. This might be dangerous for local applications, because normally adversaries are allowed to access decryption oracle for polynomial times, but our system will remain safe since the cloud server will not allow such 'decryption oracle access' for several times.

\section{Micro-Analysis for Each Operation}

We analyze the additional computation and communication overhead introduced by our system except the image process related overhead in this subsection. Due to the space limitation, we omit the evaluation results on the Android phone, whose runtime is the same order of magnitude as that on the laptop.

\section{(1) Computation Overhead}

The runtime of each operation is summarized in Table II and then the detailed analysis is presented as follows. 


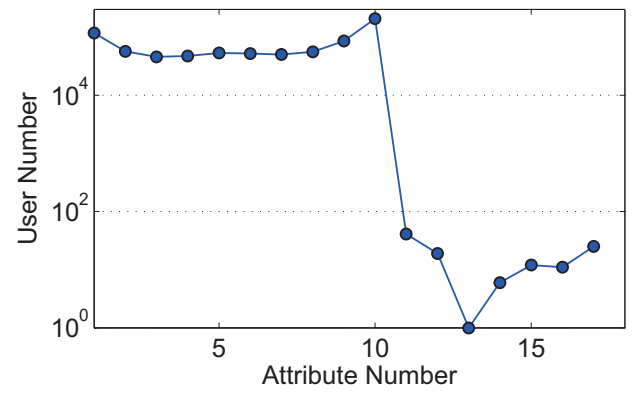

Fig. 4. Users' attribute number distribution of Tencent Weibo.

TABLE II. COMPUTATION COST FOR EACH OPERATION USING A SINGLE PC. (s) $\lambda=128, m=2$,THE SIFT-VEC IS 128-DIMENSION SIFT FEATURE VECTOR, THE FrEQ-VEC IS 1000-DIMENSION WEIGHTED FREQUENCY FEATURE VECTOR.

\begin{tabular}{|c|c|c|c|}
\hline \multicolumn{4}{|c|}{ Common Operations } \\
\hline & Mean & Min & Max \\
\hline Feature Extract & 8.7 & 1.56 & 12.03 \\
\hline Parameter Init & 0.002 & 0.002 & 0.003 \\
\hline Master Key Gen & $<0.001$ & $<0.001$ & $<0.001$ \\
\hline KA\&CS Keys Gen & 0.001 & 0.001 & 0.001 \\
\hline Triple Keys Gen & 0.002 & 0.002 & 0.003 \\
\hline Access Tree Gen & $<0.001$ & $<0.001$ & $<0.001$ \\
\hline Decrypt Distance & $<0.001$ & $<0.001$ & $<0.001$ \\
\hline \multicolumn{4}{|c|}{ Operations of PIC-sfv } \\
\hline Encrypt One SIFT-Vec & 0.034 & 0.03 & 0.047 \\
\hline Key Modification (per SIFT-Vec) & 0.052 & 0.048 & 0.063 \\
\hline Homomorphic Euclidean Distance & 0.031 & 0.029 & 0.033 \\
\hline \multicolumn{4}{|c|}{ Operation of PIC-wfv } \\
\hline & Mean & Min & $\operatorname{Max}$ \\
\hline Freq-Vec Gen & 0.70 & 0.005 & 3.84 \\
\hline Encrypt One Freq-Vec & 1.93 & 1.59 & 2.67 \\
\hline Key Modification (per Freq-Vec) & 0.29 & 0.25 & 0.85 \\
\hline Homomorphic Dot Product & 0.20 & 0.18 & 0.33 \\
\hline
\end{tabular}

Initialization: In this step, it requires a selection of system parameters for the homomorphic encryption and a selection of three random homomorphic encryption keys $k, k_{C S}, k_{K A}$, which are $4 \times 4$ matrices, such that $k=$ $k_{C S} k_{K A}$. Both operations are executed at the TP side and our results show that the system initiation takes less than $5 \mathrm{~ms}$ in total, which is negligible.

Key Generation \& Policy Announcement: In this step, it requires TP to select three random keys $k_{i}, k_{i}^{\prime}, k_{i}^{\prime \prime}$ such that $k=k_{i} k_{i}^{\prime} k_{i}^{\prime \prime}$, which costs less than 3ms. Besides, it also involves an owner's access tree generation. We evaluate the performance of our access control methods based on the profile data of Tencent Weibo [39], which is one of the largest social networking platform in China. This dataset has 2.32 million users' personal profiles, including their year of birth, gender, graduate school, profession and other tags. There are 770166 different attributes in total. Each user has 6 attributes in average and 20 attributes at most, as shown in Fig. 4. Fig. 5 illustrates the proportion of users sharing different number of common attributes. $60 \%$ users have no common attribute with others, about $20 \%$ users share one common attribute with others, and $98 \%$ users share less than four common attributes with others. Our analysis suggests that a small access tree with limited attributes (e.g. 6 attributes), is enough to narrow down

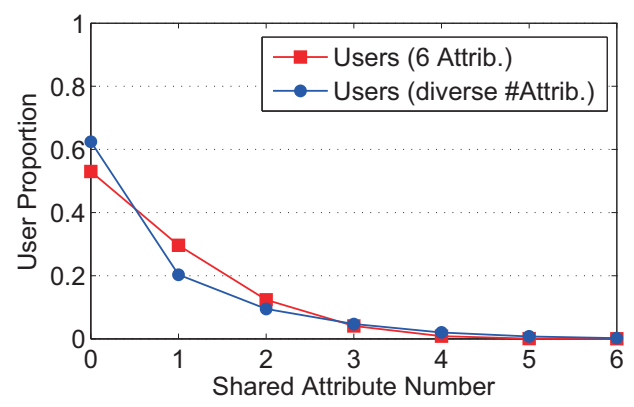

Fig. 5. The proportion of Tencent Weibo users having common attributes, except user ID which is unique for every user. The blue line is for all users with different number of attributes. The red line is for those users who have 6 attributes.

the size of authorized users (only $0.2 \%$ is valid), and its generation time is only $8 \times 10^{-3} \mathrm{~ms}$. Given the access tree, the runtime to authenticate the attributes of a querier is less than $1 \mathrm{~ms}$. So the cost for access control is negligible.

Image Upload: For PIC-sfv, the cluster construction is first executed by each owner, and the runtime increases linearly with the feature vector number (Fig. 6). For two image sets, it takes about 20 seconds to cluster feature vectors of 100 randomly selected images. Then the owner encrypts every descriptor using his key $k_{i}$. As shown in Table II, it takes $34 \mathrm{~ms}$ to encrypt each 128-dimension feature vector. The runtime to encrypt the descriptor of each image depends on the its feature vector number as depicted in Fig. 6. Fig. 7 shows the runtime distribution to encrypt one image from two images sets, which is $75 \mathrm{~s}$ in average. After the ciphertexts are sent to KA, KA conducts a key modification to alter the encryption key of the ciphertexts. As shown in Fig. 7, it takes 155 s to modify the key of an image in average. The key modification at CS side is the same operation as KA's one and thus omitted.

For PIC-wfv, based on the visual word vocabulary, the owner generates weight frequency vector for each image, whose runtime is proportional to the feature vector number of this image (Fig. 8). For two image sets, it takes $0.7 \mathrm{~s}$ per image in average. Then the owner encrypts the weighted frequency vector of each image, whose runtime is depicted in Fig 9. It takes less than 1.5s to encrypt one image. Fig. 9 also presents the time cost of key modification for each image by KA (or CS), which is about $0.29 \mathrm{~s}$. The evaluation shows that the advance scheme based on the weight frequency vector significantly improve the computation efficiency.

Privacy-preserving Image Search with Access Control: The querier first encrypts the image descriptor (SIFT feature vectors or one weighted frequency vector), whose run time is the same as the one in the image uploading (Fig. 7 and Fig. 9). The key modifications by KA and CS are also the same. Besides, in PIC-sfv, CS needs to compute the ciphertexts of all squared Euclidean distances. Using a single machine, each distance takes $31 \mathrm{~ms}$ and the whole time cost depends on the number of query feature vectors and the size of the searched vector collection in DB, i.e. $(\#$ cluster + cluster size $) \times \#$ featurevector $\times 31 \mathrm{~ms}$. In PIC-wfv, CS computes the ciphertexts of all dot prod- 


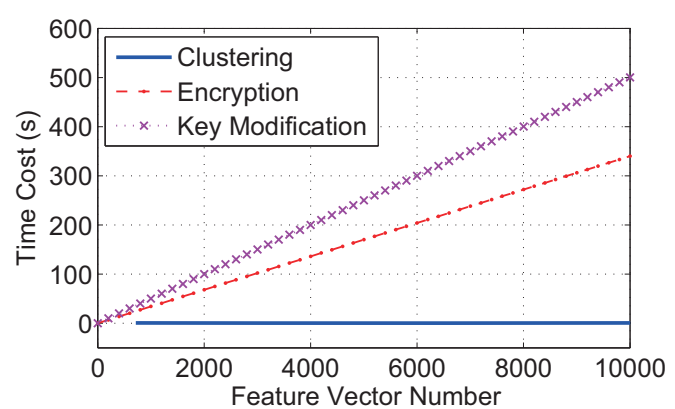

Fig. 6. Runtime of upload operation VS. SIFT feature vector Number (PIC-sfv).

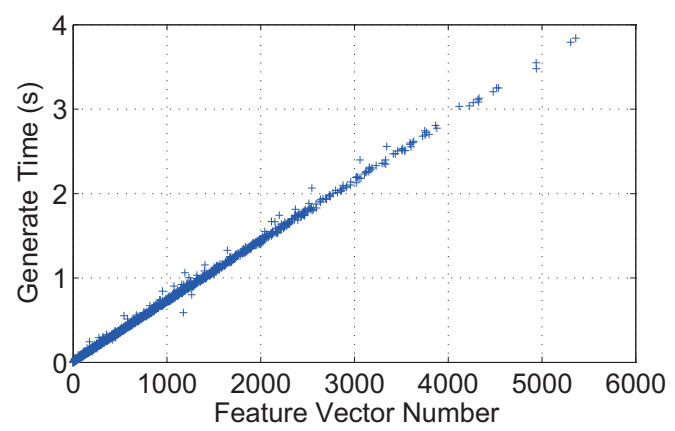

Fig. 8. 1000-dimension weighted frequency vector generation for one image VS. feature vector number of this image (PIC-wfv).

ucts, each takes $200 \mathrm{~ms}$ by one machine and the whole time cost just depends on the image collection size, i.e. (\#cluster + cluster size $) \times 200 \mathrm{~ms}$. After CS prepares all the ciphertexts, KA decrypts them to achieve the distances or dot products, and sort them to find out the NN. For both schemes, each decryption requires less than $1 \mathrm{~ms}$ and it takes about 0.5 s to decrypt 1000 distances. We use quicksort to sort the distances, but we omit the run time analysis since this is a standard sorting method. For Holiday, without MapReduce functions, using PIC-sfv each query (with more than 3000 feature vectors) averagely takes about 100 machine-hours to find the matching image among the 1491 images; using PIC-wfv each query takes about 15 machineseconds. Deploying our MapReduce implementations on the 4-node small data center, the delay is reduced to about 17 hours for PIC-sfv and reduced to 4s for PIC-wfv.

As a brief summary, both PIC-sfv and PIC-wfv have same initialization delay and similar runtime for index construction and frequency vector generation. However, PIC-wfv uses only one 1000-dimension weighted frequency vector to represent each image, while in PIC-sfv the descriptor of each image is a set of 128-dimension SIFT feature vectors. When the size of feature vectors is small, two scheme has comparable performance. But as the feature vector size increases, PIC-sfv's overhead increases linearly while PIC-wfv keeps the runtime almost a constant. Moreover, for the privacy-preserving image search, our schemes work excellently with MapReduce framework to reduce the response time.

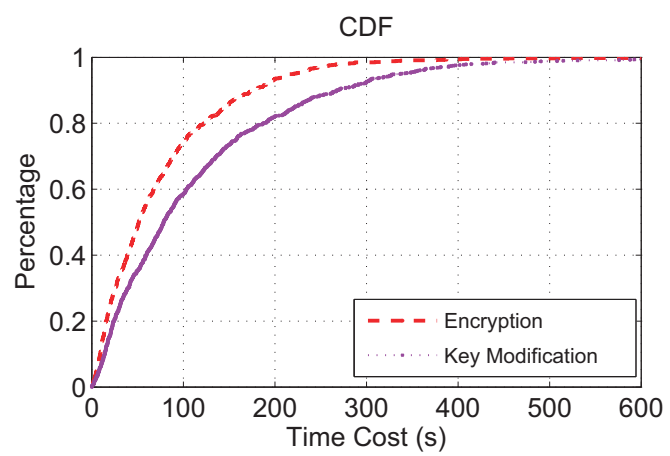

Fig. 7. CDF of runtime of upload operation for each image in two image sets (PIC-sfv).

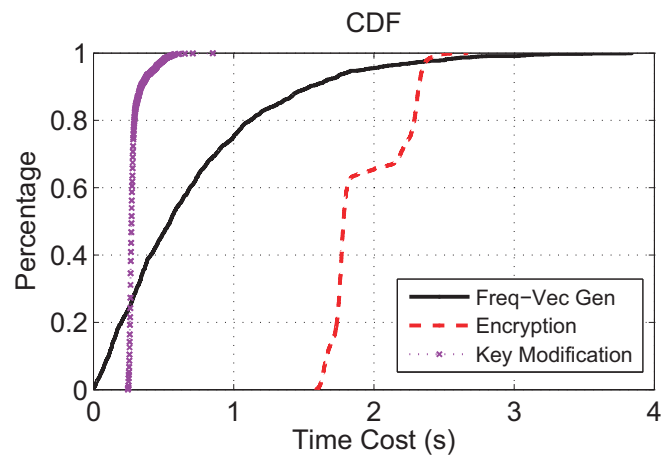

Fig. 9. CDF of runtime of upload operation for each image in two image sets (PIC-wfv).

\section{(2) Communication Overhead}

TABLE III. COMMUNiCATION COST OF EACH DATA STRUCTURE.

(KB) $\lambda=128, m=2$, THE SIFT-VEC IS 128-DIMENSION SIFT

FEATURE VeCtor, the FreQ-VEC is 1000-Dimension Weighted FREQUENCY FEATURE VECTOR.

\begin{tabular}{|c|c|c|c|}
\hline \multicolumn{4}{|c|}{ Common Data } \\
\hline & Mean & Min & $\operatorname{Max}$ \\
\hline Key & 0.84 & 0.19 & 1.4 \\
\hline Access Tree & 0.18 & 0.03 & 0.63 \\
\hline Encrypted Distance & 0.56 & 0.41 & 0.65 \\
\hline \multicolumn{4}{|c|}{ Data of PIC-sfv } \\
\hline Encrypted SIFT-Vec & 64 & 62.8 & 65.1 \\
\hline \multicolumn{4}{|c|}{ Data of PIC-wfv } \\
\hline & Mean & Min & Max \\
\hline Encrypted Freq-Vec & 580 & 578.9 & 581.2 \\
\hline
\end{tabular}

We first summarize the size of the transmitted data structure in Table III. Then we analyze the communication cost for each operation.

Initialization: TP needs to send key $k_{C S}$ and $k_{K A}$ to CS and KA respectively, and the mean size of a single key is $0.84 \mathrm{~KB}$.

Key Generation \& Policy Announcement: TP sends $k_{i}, k_{i}^{\prime} k_{i}^{\prime \prime}$ to the user $i$, CS and KA respectively, and each key's size is $0.84 \mathrm{~KB}$. Based on the user attributes of Tencent Weibo, the size of the access tree with 6 attributes is about $0.2 \mathrm{~KB}$.

Image Upload: The user informs CS of the change in the index cluster, but this is almost negligible. Main communi- 
cation overhead comes from the ciphertexts transmission. For PIC-sfv, uploading the encrypted feature vectors incurs \# feature vector $\times 64 \mathrm{~KB}$ data transmission. For PIC$\mathrm{wfv}$, the ciphertexts (encrypted weighted frequency vector) size of each image is $580 \mathrm{~KB}$, which is constant. Similarly, KA also needs to send out ciphertexts of the same size to CS.

Privacy Preserving Image Search with Access Control: First, the querier encrypts the query descriptor and sends the corresponding ciphertexts to KA, which is \# feature vector $\times 64 \mathrm{~KB}$ for PIC-sfv and $580 \mathrm{~KB}$ for PICwfv. KA also sends the same amount of ciphertexts to CS. For PIC-sfv, in the level-1 search, after CS computes the encrypted distances for all representatives, the encrypted distances are sent back to KA, the size is $0.56 \mathrm{~KB}$ each and the whole size is \#cluster $\times 0.56 \mathrm{~KB}$. During the level-2 search, CS computes the encrypted distances for all vectors within the NN's cluster, and sends them to $\mathrm{KA}$ (cluster size $\times 0.56 \mathrm{~KB})$. For PIC-wfv, similarly, CS computes and send all encrypted dot products to KA, whose size is (\#cluster + cluster size) $\times 0.56 \mathrm{~KB}$. For Holiday, the transmitted data between CS and KA during search is about $2800 \mathrm{~KB}$ for two rounds search of PIC-sfv and $43 \mathrm{~KB}$ for PIC-wfv.

\section{E. Macro-Analysis for Each Entity}

We analyze the overall computation overhead for each entity in this subsection. Similarly, we only analyze the additional overhead except that for the image processing.

Cloud Server: The computational delay at CS side during the image uploading comes from key modification. In PIC$\mathrm{sfv}$, it is \#feature vector $\times 52 \mathrm{~ms}$ (Fig. 6) and about 200s for $80 \%$ images (Fig. 7). Using PIC-wfv, it takes about $1.2 \mathrm{~s}$ for $80 \%$ images. During the image search, the computation cost of CS comes from the homomorphic distance calculation. For a single node, the cost is (\#cluster + cluster size $) \times \#$ feature vector $\times 31 \mathrm{~ms}$ using PIC-sfv and (\#cluster + cluster size $) \times 200 \mathrm{~ms}$ using PICwfv.

Key Agent: KA experiences the same delay as CS during the image upload. The computation cost of KA during search comes from decrypting all distances and ranking them to find the NN. The run time is 0.5 s to process 1000 distances.

Client: The computational delays occur during the system join, image upload and the image search for an ordinary user. The system join cost is negligible (about $3 \mathrm{~ms}$ ). In PIC-sfv, the clustering cost is negligible compared to the encryption cost, and encryption cost is \# feature vector $\times$ $34 \mathrm{~ms}$ (Fig. 6). So the computational delay of upload is about 100s for $80 \%$ images from the two image sets (Fig. 7). Similarly, for PIC-wfv, the computational delay during upload for $80 \%$ images is only about $2.2 \mathrm{~s}$ and $1.5 \mathrm{~s}$ in average(Fig. 9). During search, the client does nothing but waits for the search result from the cloud and the delay is the summation of computational delay at CS and KA.

In summary, for a querier, after providing a query image, the response time is mainly the computational delay

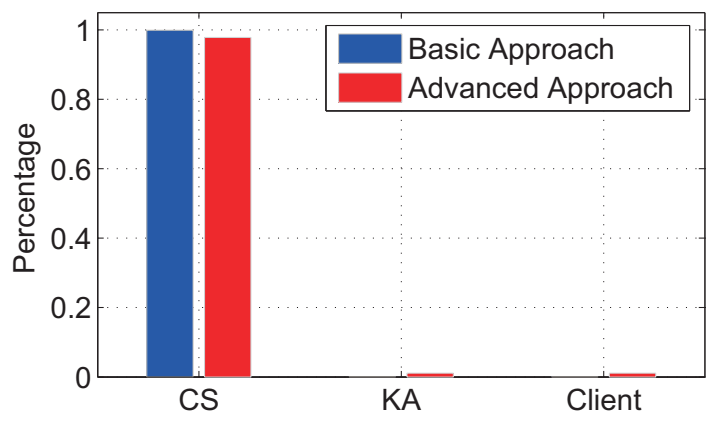

Fig. 10. Computation overhead distribution among CS, KA and client for a single query .

accumulation of image encryption, two key modifications, homomorphic distance calculation, distance decryption and ranking. When the querier can access all 1491 images of Holiday on the cloud, the average response time of each query is about 17 hours and 8 minutes for PIC-sfv and 7.21 seconds for PIC-wfv. Here the delay can be greatly reduced as the cloud scales up from 4 nodes to hundreds of nodes. Besides, compared to exiting multi-part secure computation based methods, by our approach, during the search no interaction is required for the client and $\mathbf{9 7 \%}$ computation is carried out by CS, leaving KA and clients very limited overhead (Fig. 10).

\section{F. Performance Comparison}

Compare with alternative methods. [24] has compared its cost against the well-known homomorphic encryption scheme of Gentry [40]. Gentry's scheme needs more than 900 seconds to add two 32 bit numbers, and more than 67000 seconds for the multiplication, but the cost for [24] is only $0.1 \mathrm{~ms}$ and $108 \mathrm{~ms}$ respectively. The reason is that Gentry's fully homomorphic encryption is based on the "learning with errors" (LWE) problems in lattice system, which allows users to apply as many multiplications as they want on the ciphertexts. [24] is based on number theory and group theory, which only supports a limited number of homomorphic operations, but is sufficient for our application. So, [24] is much more practical and compact. We also realize private Euclidean distance computation using a partial homomorphic encryption (Paillier encryption) in the SMC manner (e.g. the method used in [32]). Using the same computer and test images, the Paillier-based method (128-bit) takes about $0.5 \mathrm{~s}$ for feature vector encryption and $0.18 \mathrm{~s}$ for homomorphic distance computation. But in our work, they take only $0.034 \mathrm{~s}$ and $0.031 \mathrm{~s}$ respectively. The comparison shows the computation efficiency of our system.

Search Accuracy. First, we evaluate the search accuracy of our approaches according to the ground truth of 500 queries of Holiday. With $k=5$ (five nearest neighbors are fetched), the accuracy of PIC-sfv is $95.2 \%$ and of PIC$\mathrm{wfv}$ is $93.4 \%$. Here the accuracy is the success rate of 500 queries. A result is success if the returned $k$ images contain at least one image from the same scene as the query image. So, our solution achieves privacy-preserving without sacrificing the search accuracy. When a vocabulary 
is learnt, PIC-wfv provides the similarly good accuracy as PIC-sfv.

\section{Linear Search vs. Our Approaches with MapRe- duce.}

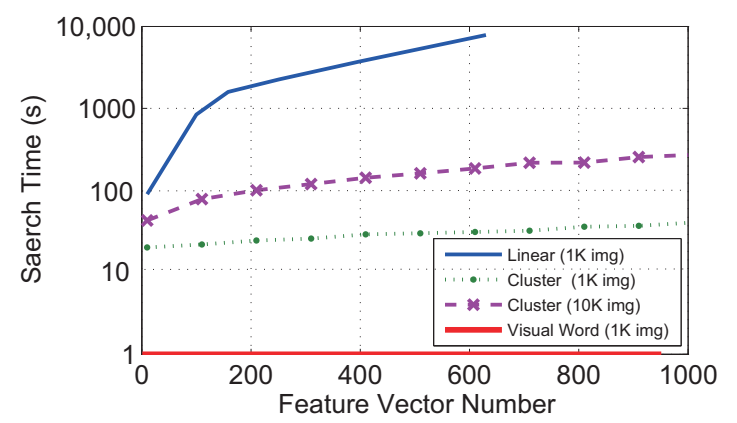

Fig. 11. Search time using different approaches VS. feature vector number of the query image. Two different size searched image collections ( $1 \mathrm{~K}$ and $10 \mathrm{~K})$ are selected randomly from Flickr1M.

We implement the SIFT feature vector based scheme and the visual word based scheme using the Hadoop MapReduce framework to accelerate the search. To study the search efficiency of different schemes, we compare the computational overhead of our two schemes using a 4-node cluster with the one of conducting a linear search using a single computer on the raw images data. The comparison is presented at Fig. 11, which shows the overhead is reduced an order of magnitude by the SIFT feature vector based approach. Furthermore, the visual word based approach keeps the overhead a second-level constant.

Then we evaluate the improvement caused by adapting our privacy-preserving search to MapReduce framework. We run the 500 queries on the encrypted Holiday data set. With a single computer, the cluster based approach takes about 100 hours for each query, and the visual word based approach takes 15 seconds for each query. When running on the 4-node small cluster with our MapReduce adaptive implementation, the runtime reduces from 100 to 17 hours and from 15 seconds to 4 s respectively. With a large cluster, the improvement will be much bigger.

With vs. Without Privacy Protection. By running the visual word based search on Holiday on the 4-node cluster in two cases, where all computation in the first case is conducted on the raw image data and all computation in the second case is conducted on ciphertexts as our system design. In the first case, the average response time for each query is $1.8 \mathrm{~s}$ and in the second case, that is $4 \mathrm{~s}$. The result shows that our system provides good protection to the image privacy with little extra overhead.

As a conclusion, leveraging the power of indexing and MapReduce, our system design improves the search performance greatly while keeping a high search accuracy and well protected privacy.

\section{RELATED WORK}

\section{A. Image Indexing and Search}

A lot of work address the problem searching for similar images, and most of them are based on the local invariant descriptors. Typically, high-dimension descriptors are extracted from the interest regions of images to represent their visual characteristics. Different types of descriptors are proposed to achieve efficient and accurate image matching, e.g., SIFT [8] and SURF [25]. The 128dimension SIFT is the most widely used descriptor for image search due to its distinctiveness and computational efficiency. An accurate approach to search similar images is to measure the distance between images' descriptors and conduct nearest neighbor search. But facing billions of high-dimension vectors, the accurate nearest neighbor search is too expensive. Various feature vector indexing approaches are designed to boost the search efficiency. A common way is using clustering to prune many clusters during the $k$-NN search, e.g., kd-tree [41] and cluster pruning [34]. Among various recent research work, extended cluster pruning [10] provides a promising way for efficient cluster construction and query processing, which outperforms the comparable solutions like p-sphere tree [42] and rank aggregation [43]. Besides clustering, Sivic and Zisserman [33] introduce the bag-of-features (BOF) for image search, which quantizes feature vectors into limited visual words using the $\mathrm{k}$-means algorithm. An image is then represented by a visual word frequency vector. Based on visual words, a lot of work speed up the search process e.g., [5]. As commercial data center get more and more popular, it is also possible to improve large-scale image search using computer clusters. MapReduce [28] provides an efficient programming framework for processing large data set in parallel. There are some work using MapReduce to accelerate the indexing and search process, e.g., [27]. However, few of the image indexing and search systems consider the privacy protection of the image owner and the querier.

\section{B. Image Privacy Protection}

To protect privacy information in the image, there are some applications simply encrypting the image or blacking out private contents, e.g., human faces. [44] removes facial characteristics from the video frame to protect the face privacy of individuals in video surveillance. P3 [45] proposes a privacy preserving photo sharing scheme by separating an image into private part and public part, and encrypts the private part directly. Many access control mechanisms are proposed to enable data owners to determine who can access their outsourced private data. For example, the data encrypted by ABE [46]-[48] can only be decrypted by the user whose attributes satisfy the access rule. [30] presents a Ciphertext-Policy Attribute-Based Encryption (CP-ABE) which keeps encrypted data confidential even if the storage server is untrusted. And the CP-ABE is improved in [49]. Those work provide privacy protection to image storage, leaving the processed image of limited use and no image search is supported for the private image. GigaSight [50] proposes an Internet system for collection of crowd-sourced video from mobile devices, which blacks out sensitive information from video frames. For search purpose, each frame is analyzed by computer vision code to obtain tags as the index. However, it cannot support image similarity based search and the indices also expose sensitive information. 


\section{Privacy Preserving Search}

Searching on encrypted (SoE) data was introduced by Song et al. [15]. It allows users to store their encrypted data on untrusted server and later search the data by keywords in a privacy-preserving manner, i.e., both the keywords and data will not be revealed. Reza et al. present a thorough discussion on the framework of searchable symmetric encryption [51]. Chang et al. improve the security and efficiency of SoE [52]. Golle et al. develop a method supporting conjunctive keyword search [16]. Lu et al. [17] propose a scheme allowing the data owner to delegate users to conducting content-level fine-grained private search and decryption. Cong, et.al. [18] extend the framework to ranked keyword search. Recently, many work are dedicated to improve the search efficiency and flexibility , e.g., [53][57]. Chen et al. [58] design a system supporting largeScale privacy-preserving mapping of human genomic sequences on hybrid clouds using a well designed hash-based mechanism. But those work mainly target text data and focus on keywords search by examining the occurrences of the searched terms (or words). They are not suitable for content-based image search since they cannot measure the distance between encrypted high-dimension feature vectors. Moreover, they usually reveal the search results to the cloud.

\section{Secure Multi-part Computation and Homomorphic En- cryption}

Privacy-preserving similarity measurement as well as search can be achieved using secure multi-party computation (SMC) [19]. SMC enables multiple parties to jointly compute a function over their inputs, while at the same time keeping these inputs private. There are some work addressing private distance computing among two parties using SMC methods [20]. Homomorphic encryption, e.g., Pallier and Elgamal, allows user to conduct the computation on the ciphertexts and obtain the ciphertext of the result, which matches the result of computation on the plaintexts. There are some work providing privacy-preserving image matching using classic homomorphic encryptions. For example, [22] and [23] allow a client privately search for a specific face image in the face image database of a server. Those methods provide privacy protection to the query image as well as the outcome of the matching algorithm, but the result is not secure against the service provider. All those privacy-preserving search mechanisms based on SMC and homomorphic encryption require rounds of online interactions with data owners during the search. Besides, computation cost of those methods are very expensive and none of them can be scaled to address large-scale image sets.

Recently, Xiao et al. propose an efficient homomorphic encryption protocol for multi-user system. [24] It is a non-circuit based symmetric-key homomorphic encryption scheme, whose security is equivalent to the large integer factorization problem. We employ this protocol to design our system.

\section{Conclusion}

We have presented a system PIC, which enables privacy-preserving content-based search on large-scale out- sourced images. With our design, the image owner can determine who is valid to search and access his images. In the image searching protocol, majority of the computationally intensive image matching jobs are outsourced to the cloud side, but the image privacy is preserved. The content of images at cloud's DB, the query result and even the query itself are kept secret to anyone else but the image owner or the querier. To further expedite the search process, we introduced the index structure and made the entire search process paralleled. We implemented our prototype system using the Hadoop MapReduce framework in a cluster of 4 computers, and our experiment results show the efficiency and applicability of our system.

\section{REFERENCES}

[1] "Boston marathon investigation," http://www.wired.com/2013/04/boston-crowdsourced.

[2] F. Korn, N. Sidiropoulos, and C. Faloutsos, "Fast nearest neighbor search in medical image databases," 1996.

[3] H. D. Tagare, C. C. Jaffe, and J. Duncan, "Medical image databases a content-based retrieval approach," Journal of the American Medical Informatics Association, vol. 4, no. 3, pp. 184-198, 1997.

[4] H. Jegou, H. Harzallah, and C. Schmid, "A contextual dissimilarity measure for accurate and efficient image search," in CVPR. IEEE, 2007.

[5] H. Jégou, M. Douze, and C. Schmid, "Improving bag-of-features for large scale image search," IJCV, vol. 87, no. 3, pp. 316-336, 2010.

[6] I. Ion, N. Sachdeva, P. Kumaraguru, and S. Čapkun, "Home is safer than the cloud!: privacy concerns for consumer cloud storage," in Proceedings of the Seventh Symposium on Usable Privacy and Security. ACM, 2011, p. 13.

[7] P. Weinzaepfel, H. Jégou, and P. Pérez, "Reconstructing an image from its local descriptors," in CVPR. IEEE, 2011.

[8] D. G. Lowe, "Distinctive image features from scale-invariant keypoints," IJCV, vol. 60, no. 2, 2004.

[9] H. Jegou, M. Douze, and C. Schmid, "Recent advances in large scale image search," in Emerging Trends in Visual Computing. Springer, 2009.

[10] G. P. Gudmundsson, B. P. Jónsson, and L. Amsaleg, "A largescale performance study of cluster-based high-dimensional indexing," in Proceedings of the international workshop on Verylarge-scale multimedia corpus, mining and retrieval. ACM, 2010, pp. 31-36.

[11] M. Daneshi and J. Guo, "Image reconstruction based on local feature descriptors," 2011.

[12] E. d'Angelo, A. Alahi, and P. Vandergheynst, "Beyond bits: Reconstructing images from local binary descriptors," in International Conference on Pattern Recognition. IEEE, 2012.

[13] "Keepsafe application," https://www.getkeepsafe.com/.

[14] D. M. Reporter, "Facebook to switch off controversial facial recognition feature following data protection concerns," http://www.dailymail.co.uk/news/article-2207098/Facebookswitch-controversial-facial-recognition-feature-following-dataprotection-concerns.html, Sep. 2012.

[15] D. X. Song, D. Wagner, and A. Perrig, "Practical techniques for searches on encrypted data," in Security and Privacy. IEEE, 2000, pp. 44-55.

[16] P. Golle, J. Staddon, and B. Waters, "Secure conjunctive keyword search over encrypted data," in Applied Cryptography and Network Security. Springer, 2004, pp. 31-45.

[17] Y. Lu and G. Tsudik, "Privacy-preserving cloud database querying," Journal of Internet Services and Information Security, vol. 1, no. 4, pp. 5-25, 2011. 
[18] C. Wang, N. Cao, K. Ren, and W. Lou, "Enabling secure and efficient ranked keyword search over outsourced cloud data," IEEE TPDS, vol. 23, no. 8, pp. 1467 - 1479, 2012.

[19] A. C.-C. Yao, "Protocols for secure computations," in FOCS, vol. 82, 1982, pp. 160-164.

[20] Y. Lindell and B. Pinkas, "A proof of yao's protocol for secure two-party computation." IACR Cryptology ePrint Archive, vol. 2004, p. 175, 2004.

[21] B. Parno, J. Howell, C. Gentry, and M. Raykova, "Pinocchio: Nearly practical verifiable computation," in SEPP. IEEE, 2013.

[22] Z. Erkin, M. Franz, J. Guajardo, S. Katzenbeisser, I. Lagendijk, and T. Toft, "Privacy-preserving face recognition," in Privacy Enhancing Technologies. Springer Berlin Heidelberg, 2009, pp. 235-253.

[23] A.-R. Sadeghi, T. Schneider, and I. Wehrenberg, "Efficient privacy-preserving face recognition," in Information, Security and Cryptology. Springer Berlin Heidelberg, 2010, pp. 229244.

[24] L. Xiao, O. Bastani, and I.-L. Yen, "An efficient homomorphic encryption protocol for multi-user systems." IACR Cryptology ePrint Archive, vol. 2012, p. 193, 2012

[25] H. Bay, A. Ess, T. Tuytelaars, and L. Van Gool, "Speededup robust features (surf)," Computer vision and image understanding, vol. 110, no. 3, pp. 346-359, 2008.

[26] H. Jegou, M. Douze, and C. Schmid, "Hamming embedding and weak geometric consistency for large scale image search," in $E C C V$. Springer, 2008.

[27] D. Moise, D. Shestakov, G. Gudmundsson, and L. Amsaleg, "Indexing and searching $100 \mathrm{~m}$ images with map-reduce," in International conference on multimedia retrieval. ACM, 2013.

[28] J. Dean and S. Ghemawat, "Mapreduce: simplified data processing on large clusters," Communications of the ACM, vol. 51, no. 1, pp. 107-113, 2008.

[29] R. Yates, "Fixed-point arithmetic: An introduction," Digital Signal Labs, vol. 81, no. 83, p. 198, 2009.

[30] J. Bethencourt, A. Sahai, and B. Waters, "Ciphertext-policy attribute-based encryption," in SEP. IEEE, 2007.

[31] T. Jung, X.-Y. Li, Z. Wan, and M. Wan, "Privacy preserving cloud data access with multi-authorities," in IEEE INFOCOM, 2013.

[32] J. Katz, A. Sahai, and B. Waters, "Predicate encryption supporting disjunctions, polynomial equations, and inner products," in Advances in Cryptology-EUROCRYPT 2008. Springer, 2008, pp. 146-162.

[33] J. Sivic and A. Zisserman, "Video google: A text retrieval approach to object matching in videos," in $I C C V$. IEEE, 2003.

[34] F. Chierichetti, A. Panconesi, P. Raghavan, M. Sozio, A. Tiberi, and E. Upfal, "Finding near neighbors through cluster pruning," in Proceedings of the twenty-sixth ACM SIGMODSIGACT-SIGART symposium on Principles of database systems. ACM, 2007, pp. 103-112.

[35] A. Sangroya, D. Serrano, and S. Bouchenak, "Mrbs: A comprehensive mapreduce benchmark suite," LIG, Grenoble, France, Research Report RR-LIG-024, 2012.

[36] - "Benchmarking dependability of mapreduce systems," in Symposium on Reliable Distributed Systems. IEEE, 2012

[37] "Pbc library," http://crypto.stanford.edu/pbc/.

[38] "Flickr1m dataset," http://www.multimediacomputing.de/wiki/Flickr1M.

[39] "Tencent weibo," http://t.qq.com/.

[40] C. Gentry, "Fully homomorphic encryption using ideal lattices," in STOC. ACM, 2009.

[41] M. Muja and D. G. Lowe, "Fast approximate nearest neighbors with automatic algorithm configuration." in $\operatorname{VISAPP}(1), 2009$, pp. 331-340.

[42] J. Goldstein and R. Ramakrishnan, "Contrast plots and psphere trees: Space vs. time in nearest neighbour searches," in $V L D B, 2000$.
[43] C. Dwork, R. Kumar, M. Naor, and D. Sivakumar, "Rank aggregation methods for the web," in International Conference on World Wide Web. ACM, 2001.

[44] E. M. Newton, L. Sweeney, and B. Malin, "Preserving privacy by de-identifying face images," TKDE, vol. 17 , no. 2 , pp. $232-$ $243,2005$.

[45] M.-R. Ra, R. Govindan, and A. Ortega, "P3: Toward privacypreserving photo sharing," in NSDI. USENIX, 2013.

[46] A. Sahai and B. Waters, "Fuzzy identity-based encryption," in Advances in Cryptology-EUROCRYPT 2005. Springer, 2005, pp. $457-473$.

[47] V. Goyal, O. Pandey, A. Sahai, and B. Waters, "Attributebased encryption for fine-grained access control of encrypted data," in Proceedings of ACM CCS, 2006.

[48] L. Guo, C. Zhang, J. Sun, and Y. Fang, "Paas: A privacypreserving attribute-based authentication system for ehealth networks," in Proceedings of IEEE ICDCS, 2012.

[49] B. Waters, "Ciphertext-policy attribute-based encryption: An expressive, efficient, and provably secure realization," in $P K C$. Springer, 2011, pp. 53-70.

[50] P. Simoens, Y. Xiao, P. Pillai, Z. Chen, K. Ha, and M. Satyanarayanan, "Scalable crowd-sourcing of video from mobile devices," in Mobisys. ACM, 2013.

[51] R. Curtmola, J. Garay, S. Kamara, and R. Ostrovsky, "Searchable symmetric encryption: improved definitions and efficient constructions," in CCS. ACM, 2006.

[52] Y.-C. Chang and M. Mitzenmacher, "Privacy preserving keyword searches on remote encrypted data," in Applied Cryptography and Network Security. Springer, 2005, pp. 442-455.

[53] C. Wang, N. Cao, K. Ren, and W. Lou, "Enabling secure and efficient ranked keyword search over outsourced cloud data," TPDS, vol. 23, no. 8, pp. 1467-1479, 2012.

[54] M. Li, S. Yu, W. Lou, and Y. T. Hou, "Toward privacyassured cloud data services with flexible search functionalities," in ICDCSW. IEEE, 2012.

[55] C. Wang, K. Ren, S. Yu, and K. M. R. Urs, "Achieving usable and privacy-assured similarity search over outsourced cloud data," in INFOCOM. IEEE, 2012, pp. 451-459.

[56] Y. Ren, Y. Chen, J. Yang, and B. Xie, "Privacy-preserving ranked multi-keyword search leveraging polynomial function in cloud computing," in Globecom. IEEE, 2014.

[57] W. Sun, S. Yu, W. Lou, Y. T. Hou, and H. Li, "Protecting your right: Attribute-based keyword search with fine-grained owner-enforced search authorization in the cloud," in IEEE INFOCOM, 2014.

[58] Y. Chen, B. Peng, X. Wang, and H. Tang, "Large-scale privacypreserving mapping of human genomic sequences on hybrid clouds." in NDSS, 2012. 